This is an author produced version of a paper published in Journal of Physics B: Atomic, Molecular and Optical Physics. This paper has been peer-reviewed but does not include the final publisher proof-corrections or journal pagination.

Citation for the published paper:

Ekman, Jörgen; Grumer, Jon; Hartman, Henrik; Jönsson, Per. (2013). A spectral study of Te $\mathrm{V}$ from MCDHF calculations. Journal of Physics $\mathrm{B}$ : Atomic, Molecular and Optical Physics, vol. 46, issue 9, p. null

URL: https://doi.org/10.1088/0953-4075/46/9/095001

Publisher: IOP Publishing

This document has been downloaded from MUEP (https://muep.mah.se) / DIVA (https://mau.diva-portal.org). 


\title{
Spectral study of Te V from MCDHF calculations
}

\author{
J. Ekman ${ }^{1}$, J. Grumer ${ }^{2}$, H. Hartman ${ }^{1,3}$, and P. Jönsson ${ }^{1}$ \\ ${ }^{1}$ Group for Materials Science and Applied Mathematics, Malmö University, 205-06 \\ Malmö, Sweden \\ ${ }^{2}$ Department of Physics, Lund University, 221-00 Lund, Sweden \\ ${ }^{3}$ Department of Astronomy and Theoretical Physics, Lund University, 221-00 Lund, \\ Sweden \\ E-mail: jorgen.ekman@mah.se
}

\begin{abstract}
State of the art multiconfiguration Dirac-Hartree-Fock calculations for Te $\mathrm{V}$ have been performed and energies, $L S$-compositions, radiative lifetimes and Landé $g_{J}$-factors for 51 odd and even parity states are presented. I addition predictions on transition rates and oscillator strengths for a multitude of transitions between these states are reported for the first time. To describe the atomic system accurately, by means of configuration interaction, valence and core-valence electron correlation effects were taken into account. Calculated energies and lifetimes of excited states agree very well with experimental data.

PACS numbers: 32.10.-f,32.30.-r,31.15.-p
\end{abstract}

Submitted to: J. Phys. B: At. Mol. Opt. Phys.

Keywords: atomic properties, atomic spectra, electronic structure 


\section{Introduction}

Belonging to the $\mathrm{Cd}$ isoelectronic sequence, four time ionized tellurium ( $\mathrm{Te} \mathrm{V}$ ) has atomic number 52 and ground state $[\mathrm{Kr}] 4 d^{10} 5 s^{2}{ }^{1} S_{0}$. A revised and extended spectrum in the 300-1230 $\AA$ region was recorded by Tauheed et al. [1] using a triggered spark source and a normal incidence spectrograph. The same authors predicted several additional levels using multiconfiguration Hartree-Fock calculations with relativistic corrections (HFR) and least squares-fitting calculations (LSF). Further on, Pinnington et al. [2] have measured lifetimes of some low-lying states by using decay-curve analysis, incorporating the adjusted normalization of decay curve (ANDC) method to correct for cascade effects, on beam-foil spectra.

The allowed and spin-forbidden $5 s^{2}{ }^{1} S_{0}-5 s 5 p{ }^{1,3} P_{1}$ transitions in Te $\mathrm{V}$ have been studied by several authors using different methods $[3,4,5]$. The most recent study is by Biémont et al. [6], who used the relativistic Hartree-Fock approach with corepolarization and also multiconfiguration Dirac-Hartree-Fock with inclusion of valence and core-valence effects. Except for the HFR energy calculations of Tauheed et al. [1] there are, to the knowledge of the authors, no theoretical studies of the more excited states.

The motivation for the present work is to improve and complement the dataset for Te V, especially for highly excited states, and to supply detailed predictions to which astronomical data can be compared. Two transitions from Te VI are in the spectra from white dwarfs $[7,8]$. The observations are very recently followed by the observation of a neutral Te I line in metal-poor stars [9]. Tellurium lines are not observed in the photospheric spectrum of the Sun; the Solar System abundance is based on meteorite studies $[10,11]$.

Heavier elements, such as Tellurium, are mainly produced through neutron capture events, and if the time-scale compared to beta-decay of the products is slow or rapid the routes are called s- or r-process, respectively. Elements are produced in either of these, or a combination, and the abundance patterns thus reveal the production properties and sites, making up the history of the creation of the elements in the object studied. Roederer et al. [9] point out the Tellurium abundance as an important discriminator between the production in the s- and r-process, partly due to the vast experimental nuclear reaction data.

\section{Computational procedure}

The many-electron wave equation can be written

$$
H \Psi=E \Psi,
$$

where the Hamiltonian $H$, in the relativistic approach using atomic units, is

$$
H_{\mathrm{DC}}=\sum_{i=1}^{N}\left(c \boldsymbol{\alpha}_{i} \cdot \boldsymbol{p}_{i}+\left(\beta_{i}-1\right) c^{2}+V_{i}^{N}\right)+\sum_{i>j}^{N} \frac{1}{r_{i j}},
$$


where $V^{N}$ is the monopole part of the electron-nucleus Coulomb interaction and $r_{i j}$ the distance between electrons $i$ and $j . \quad \alpha$ and $\beta$ are the Dirac matrices and $c$ the speed of light. The wave function $\Psi$ of the system is an eigenfunction of the total angular momentum $J^{2}$ and projection $J_{z}$ operators. In the relativistic multiconfiguration Dirac-Hartree-Fock (MCDHF) method an approximate wave function, $\Psi(\gamma J)$, for a state labeled $\gamma J$ is written as an expansion of configuration state functions (CSFs), $\Phi\left(\gamma_{i} J\right)$, with the appropriate $J$ symmetry

$$
\Psi(\gamma J)=\sum_{j=1}^{M} c_{j} \Phi\left(\gamma_{j} J\right)
$$

Here $\gamma_{i}$ represents the configuration and other quantum numbers needed to uniquely specify the state $i$. The CSFs are built from products of one-electron Dirac orbitals. The radial functions of the Dirac orbitals are unknown and should be determined on a grid. Requiring the energy computed from the multiconfiguration expansion to be stationary with respect to perturbations in the expansion coefficients leads to a matrix eigenvalue problem. The stationary condition with respect to variations in the radial functions, in turn, leads to a system of coupled integro-differential equations subject to boundary conditions at the origin and the infinity. The equations are iterated until a self-consistent solution is found [12]. The Breit interaction

$$
H_{\text {Breit }}=-\sum_{i<j}^{N}\left[\boldsymbol{\alpha}_{i} \cdot \boldsymbol{\alpha}_{j} \frac{\cos \left(\omega_{i j} r_{i j} / c\right)}{r_{i j}}+\left(\boldsymbol{\alpha}_{i} \cdot \nabla_{i}\right)\left(\boldsymbol{\alpha}_{j} \cdot \nabla_{j}\right) \frac{\cos \left(\omega_{i j} r_{i j} / c\right)-1}{\omega_{i j}^{2} r_{i j} / c^{2}}\right]
$$

as well as leading quantum electrodynamic (QED) corrections can be included in subsequent relativistic configuration interaction (RCI) calculations [13]. Calculations can be done for single levels, but also for portions of a spectrum in the extended optimal level (EOL) scheme, where optimization is on a weighted sum of energies [14]. Using the latter scheme a balanced description of a number of fine-structure states belonging to one or more configurations can be obtained in a single calculation. All calculations were performed with a new release [15] of the GRASP2K code [16].

\section{Computation of atomic properties}

Once the atomic state functions have been obtained, measurable properties like Landé $g_{J}$-factors, and transition rates can be expressed in terms of reduced matrix elements of tensor operators of different rank

$$
\left\langle\gamma P J\left\|\mathbf{T}^{(k)}\right\| \gamma^{\prime} P^{\prime} J^{\prime}\right\rangle
$$

Inserting the CSF expansions, the expression above reduces to a sum over matrix elements between CSFs. Using Racah algebra techniques these matrix elements, in turn, can be obtained as sums over radial integrals [17]. 


\subsection{Landé $g_{J}$-factors}

The Landé $g_{J}$-factors describes, to first order, the strength of the splitting of the atomic levels into magnetic sub-levels, of an atom (or ion) under the influence of an external magnetic field. In addition they give valuable information about the coupling conditions in the system [18]. The $g_{J}$-factors are given by

$$
g_{J}=\frac{2}{\sqrt{J(J+1)}}\left\langle\gamma J\left\|\sum_{j=1}^{N}\left[-i \frac{c^{2}}{\sqrt{2}} r_{j}\left(\boldsymbol{\alpha}_{j} \mathbf{C}^{(1)}(j)\right)^{(1)}+\frac{g_{s}-2}{2} \beta_{j} \boldsymbol{\Sigma}_{j}\right]\right\| \gamma J\right\rangle,(6)
$$

where $i=\sqrt{-1}$ is the imaginary unit, $\boldsymbol{\Sigma}_{j}$ the relativistic spin-matrix, and $g_{s}=2.00232$ the $g$-factor of the electron spin corrected for QED effects. The $g_{J}$-factors were calculated using the Zeeman module of GRASP2K [19].

\subsection{Transition parameters}

The transition parameters, such as rates for spontaneous decay, for electric dipole transitions between two atomic states $\gamma J$ and $\gamma^{\prime} J^{\prime}$ can be expressed in terms of the reduced matrix elements

$$
\left\langle\gamma J\left\|\mathbf{D}^{(1)}\right\| \gamma^{\prime} J^{\prime}\right\rangle
$$

where $\mathbf{D}^{(1)}$ is the electromagnetic dipole operator in length (Babushkin) or velocity (Coulomb) gauge [20]. Standard Racah algebra assumes that the atomic state functions are built from the same orthogonal radial orbital set [17]. However, this restriction can be relaxed. To compute transition matrix elements between two atomic state functions described by independently optimized orbital sets, transformations of the atomic state functions are performed in such a way that the orbital sets become biorthogonal, in which case the calculation can be handled using standard techniques [21, 22].

\section{Calculations}

\subsection{Spectrum}

The atomic state functions were determined simultaneously in extended optimal level (EOL) calculations on the weighted energy average of the fine-structure states [14]. A reference consisting of the $5 s^{2}, 5 p^{2}, 5 s 5 d, 5 s 6 d, 5 s 6 s, 5 s 7 s$, and $5 s 5 g$ configurations and $5 s 5 p, 5 s 6 p, 5 s 4 f, 5 p 5 d$, and $5 p 6 s$ configurations were chosen for the even and odd parity states, respectively. As a starting point two MCDHF calculations were performed in the EOL scheme: one calculation with CSFs describing the 22 even parity levels from the $5 s^{2}{ }^{1} S_{0}$ ground state up to $5 s 5 g{ }^{1} G_{4}$, and one calculation with CSFs describing the 29 odd parity levels from $5 s 5 p{ }^{3} P_{0}^{\circ}$ up to $5 p 6 s{ }^{1} P_{1}^{\circ}$. To correct for electron correlation effects, these calculation were followed by calculations with expansions including CSFs obtained by single(S) and double(D) excitations from, respectively, the studied even and odd state reference configurations to active sets of orbitals. Of the excitations from the even and odd state reference configurations above at most one was allowed 
from the outer $4 d$ core-shell. The $1 s, 2 s, \ldots, 4 s, 4 p$ core-shells were all kept closed. The active sets for the even and odd parity states, consisting of spectroscopic orbitals from the initial MCDHF calculations, were consecutively enlarged by adding four layers of correlation orbitals. Each layer contained orbitals with $s, p, d, f, g, h, i$ symmetries (in non-relativistic notation). Only the outermost layers of orbitals were optimized each time. The largest calculation for the even parity states included 62 relativistic orbitals and more than 278000 CSFs distributed over the $J=0,1,2,3,4,5$ symmetries. The largest calculation for the odd parity states was based on 61 relativistic orbitals and around 341000 CSFs with symmetries $J=0,1,2,3,4$. The MCDHF calculations were complemented by final RCI calculations, where the Breit interaction and leading QED corrections, self-energy and vacuum polarization, were added to the Hamiltonian.

The computational scheme described above accounts for valence and core-valence electron correlation effects. A consistent inclusion of core-core correlation effects through more than one excitation from the $4 d$ core-shell would result in an infeasible number of CSFs and thus this was not attempted. It is, however, known from other studies e.g. Harrison and Hibbert [23] that core-core effects on outer properties, like transition rates, are indeed minor and can be neglected at the present level of accuracy.

\section{Results and discussion}

\subsection{Spectrum properties}

Energy values from the spectrum calculations are displayed in Table 1. The inclusion of valence and core-valence correlation effects, built on the four layers of correlation orbitals, improves the energies dramatically, and the final energies are in very good agreement with experiment with a mean difference of $0.11 \%$. On average the calculated level energies are $190 \mathrm{~cm}^{-1}$ too low relative to the $5 s^{2}{ }^{1} S_{0}$ state and the standard deviation is around $350 \mathrm{~cm}^{-1}$. The largest discrepancies are observed for the $5 p^{2}{ }^{1} S_{0}$ level $\left(736 \mathrm{~cm}^{-1}\right.$ too high) and for the $5 s 5 p^{1} P_{1}^{\circ}$ and $5 p 5 d^{3} D_{3}^{\circ}$ levels $\left(736\right.$ and $755 \mathrm{~cm}^{-1}$ too low, respectively). Included in the comparison in Table 1 there are five predicted energy levels, denoted by asterisks, from the semi-empirical multiconfiguration Hartree Fock calculation with relativistic corrections (HFR) by Tauheed et al. 2001 [1]. The agreement with the HFR values is not as good as with experiment, reaching around $7000 \mathrm{~cm}^{-1}$ for the $5 s 4 f^{3} F_{2,3,4}^{\circ}$ and $5 s 4 f^{1} F_{3}^{\circ}$ states. There is no reason to believe that the present MCDHF calculations are less accurate for the above states compared with the other states, and thus the difference of $7000 \mathrm{~cm}^{-1}$ is most likely due to inaccurate predictions of the HFR calculations, when no semi-empirical fitting has been applied. The agreement for the $5 p 5 d^{1} F_{3}^{\circ}$ state, on the other hand, is very good.

In relativistic calculations the states are normally given in $j j$-coupling. To obtain more appropriate labels we have performed a transformation to the LSJ coupling scheme. The transformation procedure was developed by Gaigalas and co-workers $[24,25]$ and adapted for large scale calculations in the new release of the GRASP2K 
code [15]. In Table 1 we give dominant $L S$-compositions of the even and odd states. We also give the Landé $g_{J}$-factors, that provide information about the splittings of magnetic sub-levels in external magnetic fields. Many of states show heavy term mixing, especially those of the $5 p^{2}, 5 s 5 d, 5 p 5 d^{2}, 5 p 6 s$ and $5 s 5 g$ configurations. Yet, the labeling of the states is rather straightforward and agrees with the one given by Tauheed et al. [1].

Table 2 gives the calculated lifetimes for all the states, where the length gauge has been used for the electric dipole transitions. The longest lifetimes are the ones of the $5 s 5 p^{3} P^{\circ}$ terms. The extremely long lifetime of the $5 s 5 p{ }^{3} P_{2}^{\circ}$ state is due to the fact the $M 2$ transition to ground state is the only decay possibility (besides from the $M 1 / E 2$ decays to lower lying term members). In Table 2 calculated lifetimes of states belonging to eight terms are compared with values from cascade corrected beam-foil measurements by Pinnington et al. [2] and theoretical values. Values obtained from the Coulomb approximation approach are included for completeness. However, agreement of this method with experiment for systems other than alkalis is believed to be incidental and mostly the consequence of error cancellation.

The agreement between the current lifetimes and experimental lifetimes is very good. The only exception is the $5 p^{2}{ }^{1} D_{2}$ level, where the present calculated value falls in between the experimental data and the value from the Coulomb approximation. The calculated lifetimes for the $5 s 5 p{ }^{3} P_{1}^{\circ}$ and the $5 s 5 p{ }^{1} P_{1}^{\circ}$ states are $34.4 \mathrm{~ns}$ and $0.24 \mathrm{~ns}$, respectively. These lifetimes are in good agreement with the ones given by Biémont et al. [6]. The latter authors obtained $37.6 \mathrm{~ns}$ and $0.24 \mathrm{~ns}$ from a HFR calculation and $35.8 \mathrm{~ns}$ and $0.234 \mathrm{~ns}$ from an MCDHF calculation including valence and core-valence correlation. Weighted oscillator strengths and transition rates for spontaneous emission are presented in Table 3. Rates are based on computed transition energies. Length gauge has been used for the transitions. To assess the accuracy of the computed values also the ratios, $R$, between the rates in the length and velocity gauges are given. In most cases the ratio is relatively close to 1 , but for some transitions, values in the two gauges differ substantially, giving ratios far from 1 . The problematic transitions are often between states belonging to configurations differing by more than one electron. One example is the $5 p 5 d^{1} P_{1}-5 s^{2}{ }^{1} S_{0}$ transition with the rate $2.7 \times 10^{9} \mathrm{~s}^{-1}$ and $R=1.45$. Transitions between states belonging to configurations differing by more than one electron are identically zero in the independent particle model, and become allowed only due to configuration interaction effects [26]. For this reason they are very sensitive to correlation effects. It is difficult to infer the accuracy of the computed transition parameters, but weak transitions with values of $R$ far from 1 are associated with rather large uncertainties.

\section{Summary}

This work reports on extensive calculations for the level energies, lifetimes, Landé $g_{J^{-}}$ factors and transitions rates of four times ionized tellurium (Te V). Using the new release of GRASP2K [15], we describe 22 even parity states, from $5 s^{2}{ }^{1} S_{0}$ to $5 s 5 g{ }^{1} G_{4}$, 
and 29 odd parity states, from $5 s 5 p{ }^{3} P_{0}^{o}$ to $5 p 6 s{ }^{1} P_{1}^{o}$. The obtained energies are in good agreement with available experimental data [1]. For the lifetimes there is a good consistency with the beam-foil measurements by Pinnington et al. [2].

\section{Acknowledgments}

Financial support by the Swedish Research Council (VR, grant 2008-3778 and 621-20114206) is gratefully acknowledged.

\section{References}

[1] A. Tauheed, Y.N. Joshi, and A.F. Zafaran, Phys. Scripta 62 (2000) 316.

[2] E.H. Pinnington, W. Ansbacher, J.A. Kernahan, and A.S. Inamdar, J. Opt. Soc. Am. B Vol. 2, No. 2 (1985) 331.

[3] J. Migdalek and W. E. Baylis, J. Phys. B 19 (1986) 1.

[4] H.S. Chou, H.C. Chi, and K.N. Huang, Phys. Rev. A 48 (1993) 2453.

[5] C. Lavin, P. Martin, I. Martin, and J. Karwowski, Int. J. Quantum Chem. 27 (1993) 385.

[6] E. Biémont, C. Froese Fischer, M.R. Godefroid, P. Palmeri and P. Quinet, Phys. Rev. A 62 (2000) 032512-1.

[7] K. Werner, T. Rauch, E. Ringat, and J.W. Kruk, Astroph. Jour. Lett. Vol. 753:L7 (2012) 5.

[8] P. Chayer, S. Vennes, J. Dupuis, J.W. Kruk, Astroph. Jour. Lett. Vol. 630 630:L172 (2005).

[9] I.U. Roederer et al., Astroph. Jour. Lett. Vol. 747:L8 (2012) 5.

[10] M. Asplund, N. Grevesse, A.J. Sauval, P. Scott, Annu. Rev. Astron. Astrophys. 2009, 47, 481.

[11] K. Lodders, H. Palme, and H.P. Gail, 2009, in Abundances of the elements in the solar system (Springer Verlag, Berlin, Heidelberg, New York)

[12] I.P. Grant, Relativistic Quantum Theory of Atoms and Molecules, Springer, New York, 2007.

[13] B.J. McKenzie, I.P. Grant, and P.H. Norrington, Comput. Phys. Commun. 21 (1980) 233.

[14] K.G. Dyall, I.P. Grant, C.T. Johnson, F.A. Parpia, and E.P. Plummer, Comput. Phys. Commun. 55 (1989) 425.

[15] P. Jönsson, G. Gaigalas, J. Bieron, C. Froese Fischer, and I.P. Grant, Comput. Phys. Commun. (2013) accepted for publication.

[16] P. Jönsson, X. He, C. Froese Fischer, and I.P. Grant, Comput. Phys. Commun. 177 (2007) 597.

[17] G. Gaigalas, S. Fritzsche, I. P. Grant, Comput. Phys. Commun. 139 (2001) 263.

[18] C. Froese Fischer and P. Jönsson, Journal of Molecular Structure 537 (2001) 55.

[19] M. Andersson and P. Jönsson, Comput. Phys. Commun. 178 (2008) 156.

[20] I.P. Grant, J. Phys. B 7 (1974) 1458.

[21] J. Olsen , M. Godefroid, P. Jönsson, P.Å. Malmqvist, and C. Froese Fischer, Phys. Rev. E 52 (1995) 4499.

[22] P. Jönsson and C. Froese Fischer, Phys. Rev. A 57 (1998) 4967.

[23] S. Harrison and A. Hibbert Mon. Not. R. Astron. Soc. 340 (2003) 1279.

[24] G. Gaigalas, T. Žalandauskas, and Z. Rudzikas, At. Data and Nucl. Data Tables, 84 (2003) 99.

[25] G. Gaigalas, T. Žalandauskas, and S. Fritzsche, Comput. Phys. Commun. 157 (2004) 239.

[26] C. Froese Fischer, T. Brage, and P. Jönsson, 1997 Computational Atomic Structure - an MCHF approach (Institute of Physics Publishing, Bristol and Philadelphia)

[27] A. Hibbert, Nucl. Inst. Method., 202 (1982). 
Spectral study of Te $V$ from MCDHF calculations

\section{Tables and table captions}

Table 1: Calculated and experimental energy levels in $\mathrm{cm}^{-1}$. Column 2 and 3 presents receptively the $L S$ compositions and Landé $g_{J}$-factors. Excitation energies from this work are presented in column 4, and column 5 displays experimental energies or energies from HFR calculations by Tauheed et al. [1] where the latter are denoted by $*$. The last column, labelled "Diff", gives differences in energy between experiment and the largest calculation based on four layers of correlation orbitals.

\begin{tabular}{|c|c|c|c|c|c|}
\hline Level & $L S$-composition & $g_{J}$ & $E$ & Exp/HFR & Diff \\
\hline $5 s^{2}{ }^{1} S_{0}$ & $0.965 s^{2}{ }^{1} S$ & & 0 & 0 & 0 \\
\hline $5 s 5 p^{3} P_{0}^{\circ}$ & $0.985 s 5 p^{3} P^{\circ}$ & & 75218 & 75111 & -107 \\
\hline $5 s 5 p^{3} P_{1}^{\circ}$ & $0.965 s 5 p^{3} P^{\circ}+0.025 s 5 p^{1} P^{\circ}$ & 1.490 & 78076 & 78025 & -51 \\
\hline $5 s 5 p^{3} P_{2}^{\circ}$ & $0.985 s 5 p^{3} P^{\circ}$ & 1.486 & 85955 & 86004 & 49 \\
\hline $5 s 5 p{ }^{1} P_{1}^{\circ}$ & $0.935 s 5 p^{1} P^{\circ}+0.025 s 5 p^{3} P^{\circ}$ & 1.011 & 110971 & 111707 & 736 \\
\hline $5 p^{2}{ }^{3} P_{0}$ & $0.915 p^{2}{ }^{3} P+0.065 p^{2}{ }^{1} S$ & & 175905 & 176255 & 350 \\
\hline $5 p^{2}{ }^{3} P_{1}$ & $0.975 p^{2}{ }^{3} P$ & 1.501 & 181914 & 182421 & 507 \\
\hline $5 p^{2}{ }^{1} D_{2}$ & $0.545 p^{2}{ }^{1} D+0.345 p^{2}{ }^{3} P+0.105 s 5 d^{1} D$ & 1.175 & 182742 & 182804 & 62 \\
\hline $5 p^{2}{ }^{3} P_{2}$ & $0.635 p^{2}{ }^{3} P+0.265 p^{2}{ }^{1} D+0.085 s 5 d^{1} D$ & 1.324 & 192223 & 192597 & 374 \\
\hline $5 s 5 d^{3} D_{1}$ & $0.975 s 5 d^{3} D$ & 0.499 & 215295 & 215611 & 316 \\
\hline $5 p^{2}{ }^{1} S_{0}$ & $0.895 p^{2}{ }^{1} S+0.065 p^{2}{ }^{3} P$ & & 215600 & 215061 & -539 \\
\hline $5 s 5 d^{3} D_{2}$ & $0.975 s 5 d^{3} D$ & 1.167 & 215823 & 216137 & 314 \\
\hline $5 s 5 d^{3} D_{3}$ & $0.975 s 5 d^{3} D$ & 1.334 & 216764 & 216992 & 228 \\
\hline $5 s 5 d^{1} D_{2}$ & $0.785 s 5 d^{1} D+0.165 p^{2}{ }^{1} D$ & 1.001 & 231968 & 232054 & 86 \\
\hline $5 s 6 s^{3} S_{1}$ & $0.975 s 6 s^{3} S$ & 2.002 & 240886 & 240839 & -47 \\
\hline $5 s 6 s^{1} S_{0}$ & $0.975 s 6 s^{1} S$ & & 246985 & 246751 & -234 \\
\hline $4 f 5 s{ }^{3} F_{2}^{\circ}$ & $0.954 f 5 s{ }^{3} F^{\circ}+0.025 p 5 d^{3} F^{\circ}$ & 0.658 & 259007 & $251880^{*}$ & -7127 \\
\hline $4 f 5 s{ }^{3} F_{3}^{\circ}$ & $0.954 f 5 s^{3} F^{\circ}+0.025 p 5 d^{3} F^{\circ}$ & 1.083 & 259009 & $252023^{*}$ & -6986 \\
\hline $4 f 5 s^{3} F_{4}^{\circ}$ & $0.964 f 5 s^{3} F^{\circ}$ & 1.250 & 259072 & $252206^{*}$ & -6866 \\
\hline $4 f 5 s{ }^{1} F_{3}^{\circ}$ & $0.924 f 5 s{ }^{1} F^{\circ}+0.055 p 5 d{ }^{1} F^{\circ}$ & 1.000 & 262345 & $255539^{*}$ & -6806 \\
\hline $5 s 6 p^{3} P_{0}^{\circ}$ & $0.975 s 6 p^{3} P^{\circ}$ & & 273965 & 273997 & 32 \\
\hline $5 s 6 p^{3} P_{1}^{\circ}$ & $0.805 s 6 p^{3} P^{\circ}+0.175 s 6 p^{1} P^{\circ}$ & 1.414 & 274362 & 274409 & 47 \\
\hline $5 s 6 p^{3} P_{2}^{\circ}$ & $0.975 s 6 p^{3} P^{\circ}$ & 1.487 & 277420 & 277508 & 88 \\
\hline $5 s 6 p{ }^{1} P_{1}^{\circ}$ & $0.795 s 6 p{ }^{1} P^{\circ}+0.175 s 6 p^{3} P^{\circ}+0.025 p 6 s{ }^{1} P^{\circ}$ & 1.087 & 278613 & 278703 & 90 \\
\hline $5 p 5 d^{3} F_{2}^{\circ}$ & $0.745 p 5 d^{3} F^{\circ}+0.215 p 5 d^{1} D^{\circ}$ & 0.740 & 300885 & 301154 & 269 \\
\hline $5 p 5 d^{3} F_{3}^{\circ}$ & $0.885 p 5 d^{3} F^{\circ}+0.065 p 5 d^{3} D^{\circ}$ & 1.098 & 305823 & 306094 & 271 \\
\hline $5 p 5 d{ }^{1} D_{2}^{\circ}$ & $0.525 p 5 d^{1} D^{\circ}+0.185 p 5 d^{3} P^{\circ}+0.145 p 5 d^{3} F^{\circ}$ & 1.057 & 307618 & 308012 & 394 \\
\hline $5 p 5 d^{3} D_{1}^{\circ}$ & $0.735 p 5 d^{3} D^{\circ}+0.185 p 5 d^{3} P^{\circ}+0.065 p 5 d^{1} P^{\circ}$ & 0.713 & 312169 & 312698 & 529 \\
\hline
\end{tabular}


Spectral study of Te $V$ from MCDHF calculations

Table 1: Continued.

\begin{tabular}{|c|c|c|c|c|c|}
\hline Level & $L S$-composition & $g_{J}$ & $E$ & Exp/HFR & Diff \\
\hline $5 p 5 d^{3} F_{4}^{\circ}$ & $0.965 p 5 d^{3} F^{\circ}$ & 1.250 & 312523 & 312775 & 252 \\
\hline $5 p 5 d^{3} D_{2}^{\circ}$ & $0.435 p 5 d^{3} D^{\circ}+0.245 p 5 d^{3} P^{\circ}+0.235 p 5 d^{1} D^{\circ}$ & 1.155 & 315491 & 316017 & 526 \\
\hline $5 p 5 d^{3} D_{3}^{\circ}$ & $0.895 p 5 d^{3} D^{\circ}+0.075 p 5 d^{3} F^{\circ}$ & 1.311 & 319609 & 320364 & 755 \\
\hline $5 p 5 d^{3} P_{1}^{\circ}$ & $0.765 p 5 d^{3} P^{\circ}+0.205 p 5 d^{3} D^{\circ}$ & 1.297 & 321185 & 321737 & 552 \\
\hline $5 p 5 d^{3} P_{2}^{\circ}$ & $0.545 p 5 d^{3} P^{\circ}+0.405 p 5 d^{3} D^{\circ}+0.025 p 5 d^{1} D^{\circ}$ & 1.334 & 321213 & 321572 & 359 \\
\hline $5 p 5 d^{3} P_{0}^{\circ}$ & $0.965 p 5 d^{3} P^{\circ}$ & & 321239 & 321490 & 251 \\
\hline $5 s 6 d^{3} D_{1}$ & $0.975 s 6 d^{3} D$ & 0.499 & 324881 & 324880 & -1 \\
\hline $5 s 6 d^{3} D_{2}$ & $0.955 s 6 d^{3} D+0.025 s 6 d^{1} D$ & 1.163 & 325080 & 325080 & 0 \\
\hline $5 s 6 d^{3} D_{3}$ & $0.975 s 6 d^{3} D$ & 1.334 & 325548 & 325506 & -42 \\
\hline $5 s 6 d^{1} D_{2}$ & $0.935 s 6 d^{1} D+0.025 s 6 d^{3} D$ & 1.004 & 326857 & 326563 & -294 \\
\hline $5 p 5 d{ }^{1} P_{1}^{\circ}$ & $0.535 p 5 d^{1} P^{\circ}+0.215 p 6 s^{1} P^{\circ}+0.175 p 6 s{ }^{3} P^{\circ}$ & 1.084 & 333105 & 333122 & 17 \\
\hline $5 s 7 s^{3} S_{1}$ & $0.975 s 7 s^{3} S$ & 2.002 & 334487 & 334455 & -32 \\
\hline $5 p 6 s^{3} P_{0}^{\circ}$ & $0.975 p 6 s^{3} P^{\circ}$ & & 335301 & 335348 & 47 \\
\hline $5 s 7 s^{1} S_{0}$ & $0.985 s 7 s^{1} S$ & & 336320 & 336211 & -109 \\
\hline $5 p 5 d^{1} F_{3}^{\circ}$ & $0.865 p 5 d^{1} F^{\circ}+0.054 f 5 s{ }^{1} F^{\circ}+0.025 p 5 d^{3} D^{\circ}$ & 1.008 & 337546 & $338029^{*}$ & 483 \\
\hline $5 p 6 s^{3} P_{1}^{\circ}$ & $0.625 p 6 s{ }^{3} P^{\circ}+0.275 p 5 d^{1} P^{\circ}+0.045 p 6 s^{1} P^{\circ}$ & 1.315 & 337626 & 337625 & -1 \\
\hline $5 s 5 f^{3} F_{2}^{\circ}$ & $0.445 s 5 f^{3} F^{\circ}+0.225 s 6 f^{3} F^{\circ}+0.195 s 7 f^{3} F^{\circ}$ & 0.666 & 343773 & & \\
\hline $5 p 6 s^{3} P_{2}^{\circ}$ & $0.965 p 6 s^{3} P^{\circ}$ & 1.487 & 346286 & 346313 & 27 \\
\hline $5 p 6 s{ }^{1} P_{1}^{\circ}$ & $0.505 p 6 s{ }^{1} P^{\circ}+0.165 p 6 s^{3} P^{\circ}+0.135 s 9 p^{3} P^{\circ}$ & 1.091 & 348212 & 349800 & 1588 \\
\hline $5 s 5 g^{3} G_{3}$ & $0.605 s 5 g{ }^{3} G+0.175 p 5 f^{3} G+0.154 f 5 p^{3} G$ & 0.765 & 356849 & & \\
\hline $5 s 5 g{ }^{3} G_{4}$ & $0.495 s 5 g{ }^{3} G+0.245 s 5 g{ }^{1} G+0.095 p 5 f^{3} G$ & 1.042 & 357477 & & \\
\hline $5 s 5 g{ }^{3} G_{5}$ & $0.905 s 5 g{ }^{3} G+0.055 p 5 f^{3} G+0.044 f 5 p^{3} G$ & 1.200 & 358705 & & \\
\hline $5 s 5 g{ }^{1} G_{4}$ & $0.645 s 5 g^{1} G+0.285 s 5 g^{3} G+0.025 p 5 f^{1} G$ & 1.015 & 358763 & & \\
\hline
\end{tabular}


Table 2: Lifetimes, $\tau$, in units of ns. $\tau_{R C I}$ are the resulting values from this work, $\tau_{\exp }$ from beam-foil measurements by Pinnington et al. [2], and $\tau_{\text {theory }}$ are other theoretical values.

\begin{tabular}{|c|c|c|c|}
\hline Level & $\tau_{R C I}$ & $\tau_{\text {exp }}$ & $\tau_{\text {theory }}$ \\
\hline $5 p^{2}{ }^{3} P_{0}$ & 0.32 & & \\
\hline $5 p^{2}{ }^{1} S_{0}$ & 0.26 & & \\
\hline $5 s 6 s^{1} S_{0}$ & 0.14 & & \\
\hline $5 s 7 s^{1} S_{0}$ & 0.22 & & \\
\hline $5 p^{2}{ }^{3} P_{1}$ & 0.28 & & \\
\hline $5 s 5 d^{3} D_{1}$ & 0.14 & $0.15 \pm 0.05$ & $0.13^{b}$ \\
\hline $5 s 6 s^{3} S_{1}$ & 0.11 & $0.130 \pm 0.020^{a}$ & $0.14^{b}$ \\
\hline $5 s 6 d^{3} D_{1}$ & 0.57 & & \\
\hline $5 s 7 s^{3} S_{1}$ & 0.18 & & \\
\hline $5 p^{2}{ }^{1} D_{2}$ & 0.66 & $1.10 \pm 0.25$ & $0.40^{b}$ \\
\hline $5 p^{2}{ }^{3} P_{2}$ & 0.36 & & \\
\hline $5 s 5 d^{3} D_{2}$ & 0.14 & $0.17 \pm 0.03$ & $0.13^{b}$ \\
\hline $5 s 5 d{ }^{1} D_{2}$ & 0.11 & $0.15 \pm 0.04$ & $0.21^{b}$ \\
\hline $5 s 6 d^{3} D_{2}$ & 0.58 & & \\
\hline $5 s 6 d^{1} D_{2}$ & 0.54 & & \\
\hline $5 s 5 d^{3} D_{3}$ & 0.15 & $0.180 \pm 0.020$ & $0.13^{b}$ \\
\hline $5 s 6 d^{3} D_{3}$ & 0.63 & & \\
\hline $5 s 5 g{ }^{3} G_{3}$ & 0.91 & & \\
\hline $5 s 5 g{ }^{3} G_{4}$ & 0.77 & & \\
\hline $5 s 5 g{ }^{1} G_{4}$ & 0.49 & & \\
\hline $5 s 5 g{ }^{3} G_{5}$ & 0.54 & & \\
\hline $5 s 6 p^{3} P_{0}^{\circ}$ & 0.82 & & \\
\hline $5 p 5 d^{3} P_{0}^{\circ}$ & 0.11 & & \\
\hline $5 p 6 s{ }^{3} P_{0}^{\circ}$ & 0.15 & & \\
\hline $5 s 5 p^{3} P_{1}^{\circ}$ & 34.4 & $40 \pm 10$ & $37.6^{c} / 35.8^{d} / 46^{e}$ \\
\hline $5 s 5 p{ }^{1} P_{1}^{\circ}$ & 0.24 & $0.260 \pm 0.025$ & $0.23^{b} / 0.24^{c} / 0.234^{d}$ \\
\hline $5 s 6 p{ }^{3} P_{1}^{\circ}$ & 0.63 & & \\
\hline $5 s 6 p{ }^{1} P_{1}^{\circ}$ & 0.43 & & \\
\hline $5 p 5 d^{3} D_{1}^{\circ}$ & 0.90 & & \\
\hline $5 p 5 d^{3} P_{1}^{\circ}$ & 0.11 & & \\
\hline $5 p 5 d{ }^{1} P_{1}^{\circ}$ & 0.16 & & \\
\hline $5 p 6 s{ }^{3} P_{1}^{\circ}$ & 0.13 & & \\
\hline $5 p 6 s{ }^{1} P_{1}^{\circ}$ & 0.09 & & \\
\hline $4 f 5 s{ }^{3} F_{2}^{\circ}$ & 3.93 & & \\
\hline $5 s 6 p^{3} P_{2}^{\circ}$ & 0.73 & & \\
\hline
\end{tabular}


Table 2: Continued.

\begin{tabular}{llll}
\hline Level & $\tau_{R C I}$ & $\tau_{\text {exp }}$ & $\tau_{\text {theory }}$ \\
\hline $5 p 5 d^{3} F_{2}^{\circ}$ & 0.48 & & \\
$5 p 5 d d^{1} D_{2}^{\circ}$ & 0.17 & & \\
$5 p 5 d^{3} D_{2}^{\circ}$ & 0.11 & & \\
$5 p 5 d^{3} P_{2}^{\circ}$ & 0.10 & & \\
$5 s 5 f{ }^{3} F_{2}^{\circ}$ & 0.21 & & \\
$5 p 6 s{ }^{3} P_{2}^{\circ}$ & 0.13 & & \\
$4 f 5 s^{3} F_{3}^{\circ}$ & 3.99 & \\
$4 f 5 s^{1} F_{3}^{\circ}$ & 1.44 & \\
$5 p 5 d^{3} F_{3}^{\circ}$ & 0.49 & \\
$5 p 5 d^{3} D_{3}^{\circ}$ & 0.09 & \\
$5 p 5 d d^{1} F_{3}^{\circ}$ & 0.07 & \\
$4 f 5 s^{3} F_{4}^{\circ}$ & 4.11 & \\
$5 p 5 d^{3} F_{4}^{\circ}$ & 0.60 & \\
\hline \hline
\end{tabular}

${ }^{a}$ Weighted average of experimental measurements on three transitions.

${ }^{b}$ Values calculated in the Coulomb approximation, quoted from the paper by Pinnington et al. [2].

${ }^{c}$ Based on relativistic Hartree-Fock calculations from Biémont et al. [6].

${ }^{d}$ Based on multiconfiguration Dirac-Fock calculations from Biémont et al. [6].

${ }^{e}$ Based on configuration interaction calculations from Hibbert [27]. 
Table 3: Weighted oscillator strengths and rates in units of $\mathrm{s}^{-1}$ for relatively strong transitions $\left(A>10^{6} \mathrm{~s}^{-1}\right)$. Rates are based on computed transition energies. Length gauge has been used for E1 transitions. $R$ is the ratio between transition rates in length and velocity gauge. Values in square brackets represent powers of ten.

\begin{tabular}{|c|c|c|c|c|c|}
\hline Upper & Lower & $\Delta \overline{\Delta E\left(\mathrm{~cm}^{-1}\right)}$ & $\overline{g f}$ & $\bar{A}$ & $\bar{R}$ \\
\hline $5 s 5 p^{3} P_{1}^{\circ}$ & $5 s^{2}{ }^{1} S_{0}$ & 78075 & $2.143[-2]$ & $2.905[+7]$ & 0.87 \\
\hline $5 s 5 p{ }^{1} P_{1}^{\circ}$ & $5 s^{2}{ }^{1} S_{0}$ & 110971 & $1.536[+0]$ & $4.205[+9]$ & 0.92 \\
\hline $5 s 6 p^{3} P_{1}^{\circ}$ & $5 s^{2}{ }^{1} S_{0}$ & 274361 & $2.044[-2]$ & $3.421[+8]$ & 1.25 \\
\hline $5 s 6 p{ }^{1} P_{1}^{\circ}$ & $5 s^{2}{ }^{1} S_{0}$ & 278613 & $4.835[-2]$ & $8.345[+8]$ & 1.37 \\
\hline $5 p 5 d^{3} D_{1}^{\circ}$ & $5 s^{2}{ }^{1} S_{0}$ & 312168 & $4.658[-5]$ & $1.009[+6]$ & 0.79 \\
\hline $5 p 5 d^{1} P_{1}^{\circ}$ & $5 s^{2}{ }^{1} S_{0}$ & 333105 & $1.101[-3]$ & $2.717[+7]$ & 1.45 \\
\hline $5 p 6 s^{3} P_{1}^{\circ}$ & $5 s^{2}{ }^{1} S_{0}$ & 337625 & $3.065[-4]$ & $7.768[+6]$ & 0.92 \\
\hline $5 p 6 s{ }^{1} P_{1}^{\circ}$ & $5 s^{2}{ }^{1} S_{0}$ & 348211 & $1.533[-2]$ & $4.133[+8]$ & 1.27 \\
\hline $5 s 6 p^{3} P_{1}^{\circ}$ & $5 p^{2}{ }^{3} P_{0}$ & 98456 & $1.120[-3]$ & $2.414[+6]$ & 0.83 \\
\hline $5 p 5 d^{3} D_{1}^{\circ}$ & $5 p^{2}{ }^{3} P_{0}$ & 136263 & $1.943[+0]$ & $8.023[+9]$ & 0.95 \\
\hline $5 p 5 d^{3} P_{1}^{\circ}$ & $5 p^{2}{ }^{3} P_{0}$ & 145279 & $1.250[-2]$ & $5.868[+7]$ & 1.04 \\
\hline $5 p 5 d{ }^{1} P_{1}^{\circ}$ & $5 p^{2}{ }^{3} P_{0}$ & 157200 & $9.187[-2]$ & $5.048[+8]$ & 1.02 \\
\hline $5 p 6 s{ }^{3} P_{1}^{\circ}$ & $5 p^{2}{ }^{3} P_{0}$ & 161720 & $2.071[-1]$ & $1.204[+9]$ & 1.05 \\
\hline $5 p 6 s{ }^{1} P_{1}^{\circ}$ & $5 p^{2}{ }^{3} P_{0}$ & 172306 & $1.003[-2]$ & $6.620[+7]$ & 1.09 \\
\hline $5 s 6 p^{3} P_{1}^{\circ}$ & $5 p^{2}{ }^{1} S_{0}$ & 58761 & $2.248[-3]$ & $1.726[+6]$ & 0.92 \\
\hline $5 s 6 p{ }^{1} P_{1}^{\circ}$ & $5 p^{2}{ }^{1} S_{0}$ & 63013 & $9.953[-3]$ & $8.786[+6]$ & 0.91 \\
\hline $5 p 5 d^{3} P_{1}^{\circ}$ & $5 p^{2}{ }^{1} S_{0}$ & 105584 & $1.012[-3]$ & $2.509[+6]$ & 0.89 \\
\hline $5 p 5 d^{1} P_{1}^{\circ}$ & $5 p^{2}{ }^{1} S_{0}$ & 117505 & $8.029[-1]$ & $2.465[+9]$ & 0.96 \\
\hline $5 p 6 s{ }^{3} P_{1}^{\circ}$ & $5 p^{2}{ }^{1} S_{0}$ & 122025 & $5.598[-1]$ & $1.853[+9]$ & 0.96 \\
\hline $5 p 6 s{ }^{1} P_{1}^{\circ}$ & $5 p^{2}{ }^{1} S_{0}$ & 132611 & $6.662[-1]$ & $2.605[+9]$ & 0.99 \\
\hline $5 s 6 p^{3} P_{1}^{\circ}$ & $5 s 6 s^{1} S_{0}$ & 27376 & $1.622[-1]$ & $2.703[+7]$ & 0.99 \\
\hline $5 s 6 p{ }^{1} P_{1}^{\circ}$ & $5 s 6 s^{1} S_{0}$ & 31628 & $8.686[-1]$ & $1.932[+8]$ & 1.00 \\
\hline $5 p 5 d^{3} D_{1}^{\circ}$ & $5 s 6 s^{1} S_{0}$ & 65183 & $1.561[-3]$ & $1.475[+6]$ & 0.80 \\
\hline $5 p 5 d{ }^{1} P_{1}^{\circ}$ & $5 s 6 s^{1} S_{0}$ & 86120 & $2.170[-1]$ & $3.578[+8]$ & 0.94 \\
\hline $5 p 6 s{ }^{3} P_{1}^{\circ}$ & $5 s 6 s^{1} S_{0}$ & 90640 & $5.563[-2]$ & $1.016[+8]$ & 1.00 \\
\hline $5 p 6 s{ }^{1} P_{1}^{\circ}$ & $5 s 6 s^{1} S_{0}$ & 101226 & $7.626[-1]$ & $1.737[+9]$ & 0.98 \\
\hline $5 p 6 s{ }^{1} P_{1}^{\circ}$ & $5 s 7 s^{1} S_{0}$ & 11891 & $3.181[-1]$ & $1.000[+7]$ & 2.11 \\
\hline $5 p 5 d^{3} P_{0}^{\circ}$ & $5 p^{2}{ }^{3} P_{1}$ & 139324 & $5.279[-1]$ & $6.836[+9]$ & 0.96 \\
\hline $5 p 6 s{ }^{3} P_{0}^{\circ}$ & $5 p^{2}{ }^{3} P_{1}$ & 153387 & $3.207[-1]$ & $5.033[+9]$ & 1.04 \\
\hline $5 p 5 d^{3} D_{1}^{\circ}$ & $5 p^{2}{ }^{3} P_{1}$ & 130254 & $4.050[-1]$ & $1.528[+9]$ & 0.95 \\
\hline $5 p 5 d^{3} P_{1}^{\circ}$ & $5 p^{2}{ }^{3} P_{1}$ & 139270 & $1.046[+0]$ & $4.511[+9]$ & 0.95 \\
\hline $5 p 5 d^{1} P_{1}^{\circ}$ & $5 p^{2}{ }^{3} P_{1}$ & 151191 & $1.136[-2]$ & $5.773[+7]$ & 1.07 \\
\hline
\end{tabular}


Spectral study of Te $V$ from MCDHF calculations

Table 3: Continued.

\begin{tabular}{|c|c|c|c|c|c|}
\hline Upper & Lower & $\Delta E\left(\mathrm{~cm}^{-1}\right)$ & $g f$ & $A$ & $R$ \\
\hline $5 p 6 s^{3} P_{1}^{\circ}$ & $5 p^{2}{ }^{3} P_{1}$ & 155711 & $2.032[-1]$ & $1.096[+9]$ & 1.04 \\
\hline $5 p 6 s{ }^{1} P_{1}^{\circ}$ & $5 p^{2}{ }^{3} P_{1}$ & 166297 & $2.884[-2]$ & $1.773[+8]$ & 1.04 \\
\hline $5 s 6 p^{3} P_{2}^{\circ}$ & $5 p^{2}{ }^{3} P_{1}$ & 95506 & $4.124[-3]$ & $5.018[+6]$ & 0.90 \\
\hline $5 p 5 d^{3} F_{2}^{\circ}$ & $5 p^{2}{ }^{3} P_{1}$ & 118970 & $1.178[-2]$ & $2.224[+7]$ & 0.92 \\
\hline $5 p 5 d^{1} D_{2}^{\circ}$ & $5 p^{2}{ }^{3} P_{1}$ & 125704 & $1.046[+0]$ & $2.204[+9]$ & 0.96 \\
\hline $5 p 5 d^{3} D_{2}^{\circ}$ & $5 p^{2}{ }^{3} P_{1}$ & 133577 & $2.550[+0]$ & $6.070[+9]$ & 0.95 \\
\hline $5 p 5 d^{3} P_{2}^{\circ}$ & $5 p^{2}{ }^{3} P_{1}$ & 139298 & $3.145[-1]$ & $8.141[+8]$ & 0.95 \\
\hline $5 p 6 s^{3} P_{2}^{\circ}$ & $5 p^{2}{ }^{3} P_{1}$ & 164372 & $3.654[-1]$ & $1.317[+9]$ & 1.04 \\
\hline $5 s 6 p^{3} P_{0}^{\circ}$ & $5 s 5 d^{3} D_{1}$ & 58669 & $4.031[-1]$ & $9.255[+8]$ & 1.01 \\
\hline $5 p 5 d^{3} P_{0}^{\circ}$ & $5 s 5 d^{3} D_{1}$ & 105943 & $2.925[-1]$ & $2.190[+9]$ & 0.96 \\
\hline $5 p 6 s^{3} P_{0}^{\circ}$ & $5 s 5 d^{3} D_{1}$ & 120006 & $1.953[-3]$ & $1.877[+7]$ & 1.07 \\
\hline $5 s 6 p^{3} P_{1}^{\circ}$ & $5 s 5 d^{3} D_{1}$ & 59066 & $2.513[-1]$ & $1.949[+8]$ & 1.01 \\
\hline $5 s 6 p{ }^{1} P_{1}^{\circ}$ & $5 s 5 d^{3} D_{1}$ & 63318 & $5.186[-2]$ & $4.623[+7]$ & 1.01 \\
\hline $5 p 5 d^{3} D_{1}^{\circ}$ & $5 s 5 d^{3} D_{1}$ & 96873 & $1.915[-1]$ & $3.995[+8]$ & 0.92 \\
\hline $5 p 5 d^{3} P_{1}^{\circ}$ & $5 s 5 d^{3} D_{1}$ & 105889 & $5.916[-1]$ & $1.475[+9]$ & 0.94 \\
\hline $5 p 5 d^{1} P_{1}^{\circ}$ & $5 s 5 d^{3} D_{1}$ & 117810 & $8.678[-3]$ & $2.678[+7]$ & 1.03 \\
\hline $5 p 6 s{ }^{3} P_{1}^{\circ}$ & $5 s 5 d^{3} D_{1}$ & 122331 & $8.933[-4]$ & $2.972[+6]$ & 1.00 \\
\hline $4 f 5 s^{3} F_{2}^{\circ}$ & $5 s 5 d^{3} D_{1}$ & 43712 & $8.452[-1]$ & $2.154[+8]$ & 0.98 \\
\hline $5 s 6 p^{3} P_{2}^{\circ}$ & $5 s 5 d^{3} D_{1}$ & 62125 & $2.038[-2]$ & $1.049[+7]$ & 1.01 \\
\hline $5 p 5 d^{3} F_{2}^{\circ}$ & $5 s 5 d^{3} D_{1}$ & 85589 & $7.718[-1]$ & $7.542[+8]$ & 0.92 \\
\hline $5 p 5 d^{1} D_{2}^{\circ}$ & $5 s 5 d^{3} D_{1}$ & 92323 & $9.414[-2]$ & $1.070[+8]$ & 0.94 \\
\hline $5 p 5 d^{3} D_{2}^{\circ}$ & $5 s 5 d^{3} D_{1}$ & 100196 & $2.783[-1]$ & $3.727[+8]$ & 0.93 \\
\hline $5 p 5 d^{3} P_{2}^{\circ}$ & $5 s 5 d^{3} D_{1}$ & 105917 & $1.953[-1]$ & $2.923[+8]$ & 0.92 \\
\hline $5 s 5 f^{3} F_{2}^{\circ}$ & $5 s 5 d^{3} D_{1}$ & 128478 & $1.757[+0]$ & $3.869[+9]$ & 1.03 \\
\hline $5 s 6 p^{3} P_{0}^{\circ}$ & $5 s 6 s^{3} S_{1}$ & 33078 & $3.945[-1]$ & $2.879[+8]$ & 0.99 \\
\hline $5 p 5 d^{3} P_{0}^{\circ}$ & $5 s 6 s^{3} S_{1}$ & 80352 & $1.551[-3]$ & $6.679[+6]$ & 1.33 \\
\hline $5 p 6 s^{3} P_{0}^{\circ}$ & $5 s 6 s^{3} S_{1}$ & 15 & $2.765[-1]$ & $1.644[+9]$ & 0.93 \\
\hline $5 s 6 p^{3} P_{1}^{\circ}$ & $5 s 6 s^{3} S_{1}$ & 33475 & $9.936[-1]$ & $2.476[+8]$ & 0.99 \\
\hline $5 s 6 p{ }^{1} P_{1}^{\circ}$ & $5 s 6 s^{3} S_{1}$ & 37727 & $2.278[-1]$ & $7.210[+7]$ & 1.01 \\
\hline $5 p 5 d^{3} D_{1}^{\circ}$ & $5 s 6 s^{3} S_{1}$ & 71282 & $2.903[-3]$ & $3.280[+6]$ & 1.08 \\
\hline $5 p 5 d^{3} P_{1}^{\circ}$ & $5 s 6 s^{3} S_{1}$ & 80298 & $4.347[-3]$ & $6.232[+6]$ & 1.26 \\
\hline $5 p 5 d^{1} P_{1}^{\circ}$ & $5 s 6 s^{3} S_{1}$ & 92219 & $1.339[-1]$ & $2.532[+8]$ & 0.92 \\
\hline $5 p 6 s{ }^{3} P_{1}^{\circ}$ & $5 s 6 s^{3} S_{1}$ & 96739 & $5.390[-1]$ & $1.121[+9]$ & 0.93 \\
\hline $5 p 6 s{ }^{1} P_{1}^{\circ}$ & $5 s 6 s^{3} S_{1}$ & 107325 & $1.724[-1]$ & $4.416[+8]$ & 0.95 \\
\hline $5 s 6 p^{3} P_{2}^{\circ}$ & $5 s 6 s^{3} S_{1}$ & 36534 & $2.171[+0]$ & $3.865[+8]$ & 0.99 \\
\hline $5 p 5 d^{1} D_{2}^{\circ}$ & $5 s 6 s^{3} S_{1}$ & 66732 & $5.077[-3]$ & $3.016[+6]$ & 1.07 \\
\hline $5 p 5 d^{3} D_{2}^{\circ}$ & $5 s 6 s^{3} S_{1}$ & 74605 & $4.801[-3]$ & $3.565[+6]$ & 1.06 \\
\hline $5 p 5 d^{3} P_{2}^{\circ}$ & $5 s 6 s^{3} S_{1}$ & 80326 & $5.816[-3]$ & $5.007[+6]$ & 1.16 \\
\hline
\end{tabular}


Spectral study of Te $V$ from MCDHF calculations

Table 3: Continued.

\begin{tabular}{|c|c|c|c|c|c|}
\hline Upper & Lower & $\Delta E\left(\mathrm{~cm}^{-1}\right)$ & $g f$ & $A$ & $R$ \\
\hline $5 p 6 s{ }^{3} P_{2}^{\circ}$ & $5 s 6 s^{3} S_{1}$ & 105400 & $1.471[+0]$ & $2.180[+9]$ & 0.94 \\
\hline $5 p 6 s{ }^{1} P_{1}^{\circ}$ & $5 s 6 d^{3} D_{1}$ & 23330 & $9.956[-3]$ & $1.205[+6]$ & 1.59 \\
\hline $5 s 5 f^{3} F_{2}^{\circ}$ & $5 s 6 d^{3} D_{1}$ & 18892 & $1.615[+0]$ & $7.689[+7]$ & 9.58 \\
\hline $5 p 6 s{ }^{1} P_{1}^{\circ}$ & $5 s 7 s^{3} S_{1}$ & 13724 & $2.872[-2]$ & $1.203[+6]$ & 1.81 \\
\hline $5 s 6 p^{3} P_{1}^{\circ}$ & $5 p^{2}{ }^{1} D_{2}$ & 91619 & $4.978[-2]$ & $9.291[+7]$ & 1.00 \\
\hline $5 s 6 p{ }^{1} P_{1}^{\circ}$ & $5 p^{2}{ }^{1} D_{2}$ & 95871 & $2.154[-1]$ & $4.402[+8]$ & 1.02 \\
\hline $5 p 5 d^{3} D_{1}^{\circ}$ & $5 p^{2}{ }^{1} D_{2}$ & 129426 & $1.542[-3]$ & $5.744[+6]$ & 1.15 \\
\hline $5 p 5 d^{3} P_{1}^{\circ}$ & $5 p^{2}{ }^{1} D_{2}$ & 138442 & $2.432[-1]$ & $1.037[+9]$ & 0.96 \\
\hline $5 p 5 d{ }^{1} P_{1}^{\circ}$ & $5 p^{2}{ }^{1} D_{2}$ & 150363 & $4.528[-1]$ & $2.276[+9]$ & 1.00 \\
\hline $5 p 6 s{ }^{3} P_{1}^{\circ}$ & $5 p^{2}{ }^{1} D_{2}$ & 154883 & $2.114[-1]$ & $1.127[+9]$ & 1.07 \\
\hline $5 p 6 s{ }^{1} P_{1}^{\circ}$ & $5 p^{2}{ }^{1} D_{2}$ & 165469 & $2.905[-1]$ & $1.769[+9]$ & 1.06 \\
\hline $5 s 6 p^{3} P_{2}^{\circ}$ & $5 p^{2}{ }^{1} D_{2}$ & 94678 & $1.821[-3]$ & $2.177[+6]$ & 0.87 \\
\hline $5 p 5 d^{3} F_{2}^{\circ}$ & $5 p^{2}{ }^{1} D_{2}$ & 118142 & $4.917[-1]$ & $9.155[+8]$ & 0.94 \\
\hline $5 p 5 d^{1} D_{2}^{\circ}$ & $5 p^{2}{ }^{1} D_{2}$ & 124876 & $1.099[+0]$ & $2.287[+9]$ & 0.94 \\
\hline $5 p 5 d^{3} D_{2}^{\circ}$ & $5 p^{2}{ }^{1} D_{2}$ & 132749 & $3.807[-1]$ & $8.949[+8]$ & 0.93 \\
\hline $5 p 5 d^{3} P_{2}^{\circ}$ & $5 p^{2}{ }^{1} D_{2}$ & 138470 & $6.675[-1]$ & $1.707[+9]$ & 0.96 \\
\hline $5 p 6 s^{3} P_{2}^{\circ}$ & $5 p^{2}{ }^{1} D_{2}$ & 163544 & $3.870[-1]$ & $1.381[+9]$ & 1.04 \\
\hline $4 f 5 s{ }^{3} F_{3}^{\circ}$ & $5 p^{2}{ }^{1} D_{2}$ & 76266 & $3.334[-3]$ & $1.848[+6]$ & 0.89 \\
\hline $4 f 5 s{ }^{1} F_{3}^{\circ}$ & $5 p^{2}{ }^{1} D_{2}$ & 79603 & $7.624[-1]$ & $4.604[+8]$ & 0.91 \\
\hline $5 p 5 d^{3} F_{3}^{\circ}$ & $5 p^{2}{ }^{1} D_{2}$ & 123081 & $3.227[-1]$ & $4.658[+8]$ & 0.95 \\
\hline $5 p 5 d^{3} D_{3}^{\circ}$ & $5 p^{2}{ }^{1} D_{2}$ & 867 & $2.463[+0]$ & $4.397[+9]$ & 0.95 \\
\hline $5 p 5 d{ }^{1} F_{3}^{\circ}$ & $5 p^{2}{ }^{1} D_{2}$ & 803 & $1.534[+0]$ & $3.502[+9]$ & 0.96 \\
\hline $5 s 6 p^{3} P_{1}^{\circ}$ & $5 p^{2}{ }^{3} P_{2}$ & 82138 & $2.909[-2]$ & $4.364[+7]$ & 1.04 \\
\hline $5 s 6 p{ }^{1} P_{1}^{\circ}$ & $5 p^{2}{ }^{3} P_{2}$ & 86390 & $1.542[-1]$ & $2.559[+8]$ & 1.00 \\
\hline $5 p 5 d^{3} D_{1}^{\circ}$ & $5 p^{2}{ }^{3} P_{2}$ & 119946 & $1.632[-2]$ & $5.222[+7]$ & 0.96 \\
\hline $5 p 5 d^{3} P_{1}^{\circ}$ & $5 p^{2}{ }^{3} P_{2}$ & 128962 & $4.669[-1]$ & $1.727[+9]$ & 0.96 \\
\hline $5 p 5 d{ }^{1} P_{1}^{\circ}$ & $5 p^{2}{ }^{3} P_{2}$ & 140882 & $1.601[-2]$ & $7.064[+7]$ & 0.93 \\
\hline $5 p 6 s{ }^{3} P_{1}^{\circ}$ & $5 p^{2}{ }^{3} P_{2}$ & 145403 & $9.288[-2]$ & $4.366[+8]$ & 1.00 \\
\hline $5 p 6 s{ }^{1} P_{1}^{\circ}$ & $5 p^{2}{ }^{3} P_{2}$ & 155988 & $5.422[-1]$ & $2.934[+9]$ & 1.05 \\
\hline $5 s 6 p^{3} P_{2}^{\circ}$ & $5 p^{2}{ }^{3} P_{2}$ & 85197 & $1.047[-3]$ & $1.013[+6]$ & 0.80 \\
\hline $5 p 5 d^{3} F_{2}^{\circ}$ & $5 p^{2}{ }^{3} P_{2}$ & 108661 & $7.158[-2]$ & $1.127[+8]$ & 0.92 \\
\hline $5 p 5 d^{1} D_{2}^{\circ}$ & $5 p^{2}{ }^{3} P_{2}$ & 115395 & $2.674[-1]$ & $4.750[+8]$ & 0.92 \\
\hline $5 p 5 d^{3} D_{2}^{\circ}$ & $5 p^{2}{ }^{3} P_{2}$ & 123268 & $2.251[-1]$ & $4.562[+8]$ & 0.95 \\
\hline $5 p 5 d^{3} P_{2}^{\circ}$ & $5 p^{2}{ }^{3} P_{2}$ & 128989 & $2.279[+0]$ & $5.060[+9]$ & 0.95 \\
\hline $5 p 6 s^{3} P_{2}^{\circ}$ & $5 p^{2}{ }^{3} P_{2}$ & 154063 & $8.214[-1]$ & $2.601[+9]$ & 1.04 \\
\hline $4 f 5 s{ }^{1} F_{3}^{\circ}$ & $5 p^{2}{ }^{3} P_{2}$ & 70122 & $4.050[-1]$ & $1.898[+8]$ & 0.91 \\
\hline $5 p 5 d^{3} F_{3}^{\circ}$ & $5 p^{2}{ }^{3} P_{2}$ & 113600 & $1.373[-1]$ & $1.688[+8]$ & 0.94 \\
\hline $5 p 5 d{ }^{3} D_{3}^{\circ}$ & $5 p^{2}{ }^{3} P_{2}$ & 127386 & $3.212[+0]$ & $4.967[+9]$ & 0.95 \\
\hline
\end{tabular}


Spectral study of Te $V$ from MCDHF calculations

Table 3: Continued.

\begin{tabular}{|c|c|c|c|c|c|}
\hline Upper & Lower & $\Delta E\left(\mathrm{~cm}^{-1}\right)$ & $g f$ & $A$ & $R$ \\
\hline $5 p 5 d^{1} F_{3}^{\circ}$ & $5 p^{2}{ }^{3} P_{2}$ & 145323 & $1.481[+0]$ & $2.980[+9]$ & 0.96 \\
\hline $5 s 6 p^{3} P_{1}^{\circ}$ & $5 s 5 d^{3} D_{2}$ & 58539 & $7.660[-1]$ & $5.837[+8]$ & 1.01 \\
\hline $5 s 6 p{ }^{1} P_{1}^{\circ}$ & $5 s 5 d^{3} D_{2}$ & 62790 & $1.278[-1]$ & $1.121[+8]$ & 1.01 \\
\hline $5 p 5 d^{3} D_{1}^{\circ}$ & $5 s 5 d^{3} D_{2}$ & 96346 & $5.092[-1]$ & $1.051[+9]$ & 0.95 \\
\hline $5 p 5 d^{3} P_{1}^{\circ}$ & $5 s 5 d^{3} D_{2}$ & 105362 & $2.675[-1]$ & $6.603[+8]$ & 0.97 \\
\hline $5 p 5 d^{1} P_{1}^{\circ}$ & $5 s 5 d^{3} D_{2}$ & 117282 & $1.947[-2]$ & $5.956[+7]$ & 0.91 \\
\hline $5 p 6 s{ }^{3} P_{1}^{\circ}$ & $5 s 5 d^{3} D_{2}$ & 121803 & $1.946[-2]$ & $6.418[+7]$ & 0.98 \\
\hline $5 p 6 s{ }^{1} P_{1}^{\circ}$ & $5 s 5 d^{3} D_{2}$ & 132389 & $8.672[-4]$ & $3.379[+6]$ & 0.88 \\
\hline $4 f 5 s^{3} F_{2}^{\circ}$ & $5 s 5 d^{3} D_{2}$ & 43184 & $1.518[-1]$ & $3.775[+7]$ & 0.99 \\
\hline $5 s 6 p^{3} P_{2}^{\circ}$ & $5 s 5 d^{3} D_{2}$ & 61597 & $2.982[-1]$ & $1.509[+8]$ & 1.01 \\
\hline $5 p 5 d^{3} F_{2}^{\circ}$ & $5 s 5 d^{3} D_{2}$ & 85062 & $2.747[-1]$ & $2.651[+8]$ & 0.92 \\
\hline $5 p 5 d^{1} D_{2}^{\circ}$ & $5 s 5 d^{3} D_{2}$ & 91795 & $8.387[-2]$ & $9.428[+7]$ & 0.89 \\
\hline $5 p 5 d^{3} D_{2}^{\circ}$ & $5 s 5 d^{3} D_{2}$ & 99668 & $6.637[-2]$ & $8.796[+7]$ & 0.89 \\
\hline $5 p 5 d^{3} P_{2}^{\circ}$ & $5 s 5 d^{3} D_{2}$ & 105390 & $8.950[-1]$ & $1.326[+9]$ & 0.94 \\
\hline $5 s 5 f^{3} F_{2}^{\circ}$ & $5 s 5 d^{3} D_{2}$ & 127950 & $3.247[-1]$ & $7.091[+8]$ & 1.03 \\
\hline $4 f 5 s^{3} F_{3}^{\circ}$ & $5 s 5 d^{3} D_{2}$ & 43186 & $1.253[+0]$ & $2.227[+8]$ & 0.98 \\
\hline $4 f 5 s{ }^{1} F_{3}^{\circ}$ & $5 s 5 d^{3} D_{2}$ & 46522 & $8.740[-3]$ & $1.803[+6]$ & 0.98 \\
\hline $5 p 5 d^{3} F_{3}^{\circ}$ & $5 s 5 d^{3} D_{2}$ & 90000 & $1.241[+0]$ & $9.580[+8]$ & 0.92 \\
\hline $5 p 5 d^{3} D_{3}^{\circ}$ & $5 s 5 d^{3} D_{2}$ & 103786 & $6.020[-1]$ & $6.179[+8]$ & 0.92 \\
\hline $5 p 5 d^{1} F_{3}^{\circ}$ & $5 s 5 d^{3} D_{2}$ & 121723 & $2.869[-2]$ & $4.051[+7]$ & 0.93 \\
\hline $5 s 6 p^{3} P_{1}^{\circ}$ & $5 s 5 d^{1} D_{2}$ & 42393 & $1.248[-1]$ & $4.986[+7]$ & 0.99 \\
\hline $5 s 6 p{ }^{1} P_{1}^{\circ}$ & $5 s 5 d{ }^{1} D_{2}$ & 46645 & $7.651[-1]$ & $3.701[+8]$ & 0.99 \\
\hline $5 p 5 d^{3} D_{1}^{\circ}$ & $5 s 5 d^{1} D_{2}$ & 80200 & $1.928[-2]$ & $2.758[+7]$ & 1.07 \\
\hline $5 p 5 d^{1} P_{1}^{\circ}$ & $5 s 5 d{ }^{1} D_{2}$ & 01137 & $1.282[-1]$ & $2.916[+8]$ & 1.02 \\
\hline $5 p 6 s^{3} P_{1}^{\circ}$ & $5 s 5 d^{1} D_{2}$ & 05657 & $3.453[-1]$ & $8.571[+8]$ & 1.01 \\
\hline $5 p 6 s{ }^{1} P_{1}^{\circ}$ & $5 s 5 d^{1} D_{2}$ & 116243 & $4.013[-1]$ & $1.206[+9]$ & 1.02 \\
\hline $5 p 5 d^{3} F_{2}^{\circ}$ & $5 s 5 d^{1} D_{2}$ & 6 & $3.470[-2]$ & $2.199[+7]$ & 0.89 \\
\hline $5 p 5 d^{1} D_{2}^{\circ}$ & $5 s 5 d^{1} D_{2}$ & 50 & $1.281[-1]$ & $9.783[+7]$ & 0.90 \\
\hline $5 p 5 d^{3} D_{2}^{\circ}$ & $5 s 5 d{ }^{1} D_{2}$ & 23 & $5.742[-2]$ & $5.344[+7]$ & 0.91 \\
\hline $5 p 5 d^{3} P_{2}^{\circ}$ & $5 s 5 d^{1} D_{2}$ & 89244 & $1.213[-3]$ & $1.289[+6]$ & 0.83 \\
\hline $5 s 5 f^{3} F_{2}^{\circ}$ & $5 s 5 d^{1} D_{2}$ & 111805 & $9.220[-4]$ & $1.538[+6]$ & 0.99 \\
\hline $5 p 6 s{ }^{3} P_{2}^{\circ}$ & $5 s 5 d^{1} D_{2}$ & 114318 & $4.677[-3]$ & $8.154[+6]$ & 1.00 \\
\hline $4 f 5 s{ }^{1} F_{3}^{\circ}$ & $5 s 5 d{ }^{1} D_{2}$ & 30377 & $4.642[-1]$ & $4.081[+7]$ & 1.19 \\
\hline $5 p 5 d^{3} F_{3}^{\circ}$ & $5 s 5 d{ }^{1} D_{2}$ & 73855 & $1.230[-1]$ & $6.391[+7]$ & 0.95 \\
\hline $5 p 5 d^{3} D_{3}^{\circ}$ & $5 s 5 d{ }^{1} D_{2}$ & 87641 & $3.020[-2]$ & $2.211[+7]$ & 0.97 \\
\hline $5 p 5 d^{1} F_{3}^{\circ}$ & $5 s 5 d^{1} D_{2}$ & 105577 & $7.268[+0]$ & $7.720[+9]$ & 0.96 \\
\hline $5 p 6 s{ }^{1} P_{1}^{\circ}$ & $5 s 6 d^{3} D_{2}$ & 23132 & $7.692[-2]$ & $9.152[+6]$ & 1.52 \\
\hline $5 s 5 f^{3} F_{2}^{\circ}$ & $5 s 6 d^{3} D_{2}$ & 18693 & $2.880[-1]$ & $1.343[+7]$ & 10.21 \\
\hline
\end{tabular}


Spectral study of Te $V$ from MCDHF calculations

Table 3: Continued.

\begin{tabular}{|c|c|c|c|c|c|}
\hline Upper & Lower & $\Delta E\left(\mathrm{~cm}^{-1}\right)$ & $g f$ & $A$ & $R$ \\
\hline $5 p 6 s^{1} P_{1}^{\circ}$ & $5 s 6 d^{1} D_{2}$ & 21355 & $4.428[-1]$ & $4.489[+7]$ & 1.46 \\
\hline $5 s 6 p^{3} P_{2}^{\circ}$ & $5 s 5 d^{3} D_{3}$ & 60656 & $1.648[+0]$ & $8.087[+8]$ & 1.03 \\
\hline $5 p 5 d^{1} D_{2}^{\circ}$ & $5 s 5 d^{3} D_{3}$ & 90854 & $4.226[-1]$ & $4.654[+8]$ & 0.95 \\
\hline $5 p 5 d^{3} D_{2}^{\circ}$ & $5 s 5 d^{3} D_{3}$ & 98727 & $6.478[-1]$ & $8.423[+8]$ & 0.93 \\
\hline $5 p 5 d^{3} P_{2}^{\circ}$ & $5 s 5 d^{3} D_{3}$ & 104449 & $2.944[-1]$ & $4.285[+8]$ & 0.90 \\
\hline $5 s 5 f^{3} F_{2}^{\circ}$ & $5 s 5 d^{3} D_{3}$ & 127009 & $9.239[-3]$ & $1.988[+7]$ & 1.03 \\
\hline $5 p 6 s^{3} P_{2}^{\circ}$ & $5 s 5 d^{3} D_{3}$ & 129522 & $9.085[-4]$ & $2.033[+6]$ & 0.48 \\
\hline $4 f 5 s^{3} F_{3}^{\circ}$ & $5 s 5 d^{3} D_{3}$ & 42245 & $1.504[-1]$ & $2.558[+7]$ & 0.99 \\
\hline $5 p 5 d^{3} F_{3}^{\circ}$ & $5 s 5 d^{3} D_{3}$ & 89059 & $5.337[-1]$ & $4.033[+8]$ & 0.97 \\
\hline $5 p 5 d^{3} D_{3}^{\circ}$ & $5 s 5 d^{3} D_{3}$ & 102845 & $1.151[+0]$ & $1.161[+9]$ & 0.93 \\
\hline $5 p 5 d^{1} F_{3}^{\circ}$ & $5 s 5 d^{3} D_{3}$ & 120782 & $6.302[-2]$ & $8.761[+7]$ & 0.89 \\
\hline $4 f 5 s^{3} F_{4}^{\circ}$ & $5 s 5 d^{3} D_{3}$ & 42308 & $1.835[+0]$ & $2.435[+8]$ & 0.98 \\
\hline $5 p 5 d^{3} F_{4}^{\circ}$ & $5 s 5 d^{3} D_{3}$ & 95759 & $2.450[+0]$ & $1.665[+9]$ & 0.94 \\
\hline $5 p 6 s^{3} P_{2}^{\circ}$ & $5 s 6 d^{3} D_{3}$ & 20737 & $4.394[-2]$ & $2.521[+6]$ & 1.62 \\
\hline $5 p^{2}{ }^{3} P_{1}$ & $5 s 5 p^{3} P_{0}^{\circ}$ & 106695 & $5.486[-1]$ & $1.389[+9]$ & 0.94 \\
\hline $5 s 5 d^{3} D_{1}$ & $5 s 5 p^{3} P_{0}^{\circ}$ & 1400 & $9.571[-1]$ & $4.176[+9]$ & 0.96 \\
\hline $5 s 6 s^{3} S_{1}$ & $5 s 5 p^{3} P_{0}^{\circ}$ & 1656 & $1.573[-1]$ & $9.601[+8]$ & 1.04 \\
\hline $5 s 6 d^{3} D_{1}$ & $5 s 5 p^{3} P_{0}^{\circ}$ & 249662 & $1.027[-2]$ & $1.423[+8]$ & 0.69 \\
\hline $5 s 7 s^{3} S_{1}$ & $5 s 5 p^{3} P_{0}^{\circ}$ & 259269 & $2.803[-2]$ & $4.190[+8]$ & 1.06 \\
\hline $5 s 6 d^{3} D_{1}$ & $5 s 6 p^{3} P_{0}^{\circ}$ & 50916 & $1.291[+0]$ & $7.443[+8]$ & 1.00 \\
\hline $5 s 7 s^{3} S_{1}$ & $5 s 6 p^{3} P_{0}^{\circ}$ & 60522 & $2.543[-1]$ & $2.071[+8]$ & 1.03 \\
\hline $5 p^{2}{ }^{3} P_{0}$ & $5 s 5 p{ }^{3} P_{1}^{\circ}$ & 97829 & $4.884[-1]$ & $3.118[+9]$ & 0.94 \\
\hline $5 p^{2}{ }^{1} S_{0}$ & $5 s 5 p{ }^{3} P_{1}^{\circ}$ & 137524 & $6.803[-3]$ & $8.582[+7]$ & 0.91 \\
\hline $5 s 6 s{ }^{1} S_{0}$ & $5 s 5 p{ }^{3} P_{1}^{\circ}$ & 168909 & $5.694[-3]$ & $1.084[+8]$ & 1.03 \\
\hline $5 s 7 s{ }^{1} S_{0}$ & $5 s 5 p{ }^{3} P_{1}^{\circ}$ & 258244 & $1.147[-3]$ & $5.104[+7]$ & 1.02 \\
\hline $5 p^{2}{ }^{3} P_{1}$ & $5 s 5 p{ }^{3} P_{1}^{\circ}$ & 103837 & $3.869[-1]$ & $9.276[+8]$ & 0.94 \\
\hline $5 s 5 d^{3} D_{1}$ & $5 s 5 p{ }^{3} P_{1}^{\circ}$ & 137218 & $7.111[-1]$ & $2.977[+9]$ & 0.96 \\
\hline $5 s 6 s{ }^{3} S_{1}$ & $5 s 5 p{ }^{3} P_{1}^{\circ}$ & 162810 & $4.853[-1]$ & $2.860[+9]$ & 1.04 \\
\hline $5 s 6 d^{3} D_{1}$ & $5 s 5 p{ }^{3} P_{1}^{\circ}$ & 246804 & $6.319[-3]$ & $8.558[+7]$ & 0.67 \\
\hline $5 s 7 s{ }^{3} S_{1}$ & $5 s 5 p{ }^{3} P_{1}^{\circ}$ & 256411 & $8.470[-2]$ & $1.238[+9]$ & 1.05 \\
\hline $5 p^{2}{ }^{1} D_{2}$ & $5 s 5 p^{3} P_{1}^{\circ}$ & 104666 & $3.576[-1]$ & $5.226[+8]$ & 0.94 \\
\hline $5 p^{2}{ }^{3} P_{2}$ & $5 s 5 p{ }^{3} P_{1}^{\circ}$ & 114146 & $3.787[-1]$ & $6.582[+8]$ & 0.94 \\
\hline $5 s 5 d^{3} D_{2}$ & $5 s 5 p{ }^{3} P_{1}^{\circ}$ & 137746 & $2.129[+0]$ & $5.390[+9]$ & 0.95 \\
\hline $5 s 5 d^{1} D_{2}$ & $5 s 5 p{ }^{3} P_{1}^{\circ}$ & 153892 & $5.170[-2]$ & $1.633[+8]$ & 0.95 \\
\hline $5 s 6 d^{3} D_{2}$ & $5 s 5 p{ }^{3} P_{1}^{\circ}$ & 247003 & $2.323[-2]$ & $1.891[+8]$ & 0.69 \\
\hline $5 s 6 d^{1} D_{2}$ & $5 s 5 p{ }^{3} P_{1}^{\circ}$ & 248780 & $9.680[-4]$ & $7.993[+6]$ & 0.98 \\
\hline $5 p^{2}{ }^{3} P_{0}$ & $5 s 5 p{ }^{1} P_{1}^{\circ}$ & 64933 & $5.565[-3]$ & $1.565[+7]$ & 0.99 \\
\hline $5 p^{2}{ }^{1} S_{0}$ & $5 s 5 p{ }^{1} P_{1}^{\circ}$ & 104629 & $5.251[-1]$ & $3.834[+9]$ & 0.96 \\
\hline
\end{tabular}


Spectral study of Te $V$ from MCDHF calculations

Table 3: Continued.

\begin{tabular}{|c|c|c|c|c|c|}
\hline Upper & Lower & $\Delta E\left(\mathrm{~cm}^{-1}\right)$ & $g f$ & $A$ & $R$ \\
\hline $5 s 6 s^{1} S_{0}$ & $5 s 5 p^{1} P_{1}^{\circ}$ & 136014 & $5.589[-1]$ & $6.896[+9]$ & 1.03 \\
\hline $5 s 7 s{ }^{1} S_{0}$ & $5 s 5 p{ }^{1} P_{1}^{\circ}$ & 225349 & $8.249[-2]$ & $2.794[+9]$ & 1.02 \\
\hline $5 p^{2}{ }^{3} P_{1}$ & $5 s 5 p{ }^{1} P_{1}^{\circ}$ & 70942 & $5.243[-3]$ & $5.867[+6]$ & 0.89 \\
\hline $5 s 5 d^{3} D_{1}$ & $5 s 5 p{ }^{1} P_{1}^{\circ}$ & 104323 & $1.501[-2]$ & $3.633[+7]$ & 0.93 \\
\hline $5 s 6 s^{3} S_{1}$ & $5 s 5 p{ }^{1} P_{1}^{\circ}$ & 129914 & $1.544[-2]$ & $5.794[+7]$ & 1.02 \\
\hline $5 s 7 s^{3} S_{1}$ & $5 s 5 p{ }^{1} P_{1}^{\circ}$ & 223516 & $1.882[-3]$ & $2.091[+7]$ & 1.02 \\
\hline $5 p^{2}{ }^{1} D_{2}$ & $5 s 5 p{ }^{1} P_{1}^{\circ}$ & 71770 & $3.005[-1]$ & $2.065[+8]$ & 0.93 \\
\hline $5 p^{2}{ }^{3} P_{2}$ & $5 s 5 p{ }^{1} P_{1}^{\circ}$ & 81251 & $2.077[-1]$ & $1.829[+8]$ & 0.93 \\
\hline $5 s 5 d^{3} D_{2}$ & $5 s 5 p{ }^{1} P_{1}^{\circ}$ & 104851 & $1.622[-2]$ & $2.379[+7]$ & 0.99 \\
\hline $5 s 5 d^{1} D_{2}$ & $5 s 5 p{ }^{1} P_{1}^{\circ}$ & 120996 & $4.752[+0]$ & $9.280[+9]$ & 0.95 \\
\hline $5 s 6 d^{3} D_{2}$ & $5 s 5 p{ }^{1} P_{1}^{\circ}$ & 214108 & $1.096[-3]$ & $6.701[+6]$ & 0.90 \\
\hline $5 s 6 d^{1} D_{2}$ & $5 s 5 p{ }^{1} P_{1}^{\circ}$ & 215885 & $6.339[-2]$ & $3.941[+8]$ & 0.78 \\
\hline $5 s 7 s^{1} S_{0}$ & $5 s 6 p^{3} P_{1}^{\circ}$ & 61958 & $1.253[-1]$ & $3.208[+8]$ & 1.02 \\
\hline $5 s 6 d^{3} D_{1}$ & $5 s 6 p^{3} P_{1}^{\circ}$ & 50519 & $7.925[-1]$ & $4.497[+8]$ & 1.00 \\
\hline $5 s 7 s^{3} S_{1}$ & $5 s 6 p^{3} P_{1}^{\circ}$ & 60125 & $6.398[-1]$ & $5.142[+8]$ & 1.03 \\
\hline $5 s 6 d^{3} D_{2}$ & $5 s 6 p^{3} P_{1}^{\circ}$ & 50717 & $2.751[+0]$ & $9.441[+8]$ & 1.00 \\
\hline $5 s 6 d^{1} D_{2}$ & $5 s 6 p^{3} P_{1}^{\circ}$ & 52494 & $3.057[-1]$ & $1.124[+8]$ & 0.99 \\
\hline $5 s 7 s^{1} S_{0}$ & $5 s 6 p{ }^{1} P_{1}^{\circ}$ & 57706 & $6.681[-1]$ & $1.484[+9]$ & 1.02 \\
\hline $5 s 6 d^{3} D_{1}$ & $5 s 6 p{ }^{1} P_{1}^{\circ}$ & 46267 & $1.736[-1]$ & $8.262[+7]$ & 1.00 \\
\hline $5 s 7 s^{3} S_{1}$ & $5 s 6 p{ }^{1} P_{1}^{\circ}$ & 55873 & $1.517[-1]$ & $1.053[+8]$ & 1.03 \\
\hline $5 s 6 d^{3} D_{2}$ & $5 s 6 p{ }^{1} P_{1}^{\circ}$ & 56 & $1.858[-1]$ & $5.351[+7]$ & 1.01 \\
\hline $5 s 6 d^{1} D_{2}$ & $5 s 6 p{ }^{1} P_{1}^{\circ}$ & 48243 & $3.426[+0]$ & $1.064[+9]$ & 1.00 \\
\hline $5 p^{2}{ }^{3} P_{1}$ & $5 s 5 p{ }^{3} P_{2}^{\circ}$ & 95958 & $5.844[-1]$ & $1.196[+9]$ & 0.94 \\
\hline $5 s 5 d^{3} D_{1}$ & $5 s 5 p^{3} P_{2}^{\circ}$ & 129339 & $4.948[-2]$ & $1.841[+8]$ & 0.96 \\
\hline $5 s 6 s^{3} S_{1}$ & $5 s 5 p^{3} P_{2}^{\circ}$ & 154930 & $9.305[-1]$ & $4.966[+9]$ & 1.03 \\
\hline $5 s 6 d^{3} D_{1}$ & $5 s 5 p^{3} P_{2}^{\circ}$ & 238925 & $2.547[-4]$ & $3.233[+6]$ & 0.61 \\
\hline $5 s 7 s^{3} S_{1}$ & $5 s 5 p^{3} P_{2}^{\circ}$ & 31 & $1.520[-1]$ & $2.087[+9]$ & 1.05 \\
\hline $5 p^{2}{ }^{1} D_{2}$ & $5 s 5 p^{3} P_{2}^{\circ}$ & 86 & $6.301[-1]$ & $7.874[+8]$ & 0.94 \\
\hline $5 p^{2}{ }^{3} P_{2}$ & $5 s 5 p{ }^{3} P_{2}^{\circ}$ & 106267 & $1.284[+0]$ & $1.934[+9]$ & 0.94 \\
\hline $5 s 5 d^{3} D_{2}$ & $5 s 5 p{ }^{3} P_{2}^{\circ}$ & 129867 & $7.472[-1]$ & $1.681[+9]$ & 0.96 \\
\hline $5 s 5 d^{1} D_{2}$ & $5 s 5 p{ }^{3} P_{2}^{\circ}$ & 146012 & $2.153[-3]$ & $6.123[+6]$ & 0.92 \\
\hline $5 s 6 d^{3} D_{2}$ & $5 s 5 p^{3} P_{2}^{\circ}$ & 239124 & $4.140[-3]$ & $3.158[+7]$ & 0.63 \\
\hline $5 s 6 d{ }^{1} D_{2}$ & $5 s 5 p^{3} P_{2}^{\circ}$ & 240901 & $2.020[-4]$ & $1.564[+6]$ & 0.73 \\
\hline $5 s 5 d^{3} D_{3}$ & $5 s 5 p{ }^{3} P_{2}^{\circ}$ & 130808 & $4.051[+0]$ & $6.604[+9]$ & 0.95 \\
\hline $5 s 6 d^{3} D_{3}$ & $5 s 5 p^{3} P_{2}^{\circ}$ & 239592 & $3.073[-2]$ & $1.681[+8]$ & 0.65 \\
\hline $5 s 6 d^{3} D_{1}$ & $4 f 5 s{ }^{3} F_{2}^{\circ}$ & 65873 & $2.250[-1]$ & $2.171[+8]$ & 1.09 \\
\hline $5 s 6 d^{3} D_{2}$ & $4 f 5 s{ }^{3} F_{2}^{\circ}$ & 66072 & $3.835[-2]$ & $2.234[+7]$ & 1.09 \\
\hline $5 s 6 d^{1} D_{2}$ & $4 f 5 s^{3} F_{2}^{\circ}$ & 67849 & $1.646[-3]$ & $1.011[+6]$ & 1.15 \\
\hline
\end{tabular}


Spectral study of Te $V$ from MCDHF calculations

Table 3: Continued.

\begin{tabular}{|c|c|c|c|c|c|}
\hline Upper & Lower & $\Delta E\left(\mathrm{~cm}^{-1}\right)$ & $g f$ & $A$ & $R$ \\
\hline $5 s 5 g^{3} G_{3}$ & $4 f 5 s^{3} F_{2}^{\circ}$ & 97841 & $7.601[-1]$ & $6.934[+8]$ & 0.76 \\
\hline $5 s 6 d^{3} D_{1}$ & $5 s 6 p^{3} P_{2}^{\circ}$ & 47460 & $6.435[-2]$ & $3.223[+7]$ & 1.00 \\
\hline $5 s 7 s^{3} S_{1}$ & $5 s 6 p^{3} P_{2}^{\circ}$ & 57067 & $1.419[+0]$ & $1.027[+9]$ & 1.03 \\
\hline $5 s 6 d^{3} D_{2}$ & $5 s 6 p^{3} P_{2}^{\circ}$ & 47659 & $9.511[-1]$ & $2.882[+8]$ & 1.00 \\
\hline $5 s 6 d{ }^{1} D_{2}$ & $5 s 6 p^{3} P_{2}^{\circ}$ & 49436 & $2.229[-2]$ & $7.266[+6]$ & 1.01 \\
\hline $5 s 6 d^{3} D_{3}$ & $5 s 6 p^{3} P_{2}^{\circ}$ & 48128 & $5.522[+0]$ & $1.219[+9]$ & 1.00 \\
\hline $5 s 5 g{ }^{3} G_{3}$ & $5 p 5 d^{3} F_{2}^{\circ}$ & 55964 & $9.558[-1]$ & $2.853[+8]$ & 0.69 \\
\hline $5 s 7 s{ }^{3} S_{1}$ & $5 p 5 d{ }^{1} D_{2}^{\circ}$ & 26869 & $7.216[-3]$ & $1.158[+6]$ & 0.94 \\
\hline $5 s 5 g^{3} G_{3}$ & $5 p 5 d{ }^{1} D_{2}^{\circ}$ & 49230 & $3.202[-2]$ & $7.394[+6]$ & 0.68 \\
\hline $5 s 5 g^{3} G_{3}$ & $5 s 5 f^{3} F_{2}^{\circ}$ & 13075 & $1.078[+0]$ & $1.756[+7]$ & 0.36 \\
\hline $5 s 6 d^{3} D_{2}$ & $4 f 5 s^{3} F_{3}^{\circ}$ & 66070 & $3.177[-1]$ & $1.850[+8]$ & 1.08 \\
\hline $5 s 6 d^{1} D_{2}$ & $4 f 5 s^{3} F_{3}^{\circ}$ & 67848 & $1.113[-2]$ & $6.835[+6]$ & 1.07 \\
\hline $5 s 6 d^{3} D_{3}$ & $4 f 5 s^{3} F_{3}^{\circ}$ & 66539 & $3.764[-2]$ & $1.588[+7]$ & 1.09 \\
\hline $5 s 5 g^{3} G_{3}$ & $4 f 5 s{ }^{3} F_{3}^{\circ}$ & 97840 & $7.612[-3]$ & $6.943[+6]$ & 0.38 \\
\hline $5 s 5 g^{3} G_{4}$ & $4 f 5 s^{3} F_{3}^{\circ}$ & 98468 & $1.065[+0]$ & $7.652[+8]$ & 0.82 \\
\hline $5 s 5 g{ }^{1} G_{4}$ & $4 f 5 s{ }^{3} F_{3}^{\circ}$ & 99754 & $6.350[-1]$ & $4.683[+8]$ & 0.91 \\
\hline $5 s 6 d^{3} D_{2}$ & $4 f 5 s{ }^{1} F_{3}^{\circ}$ & 62734 & $2.018[-2]$ & $1.060[+7]$ & 1.10 \\
\hline $5 s 6 d^{1} D_{2}$ & $4 f 5 s{ }^{1} F_{3}^{\circ}$ & 64511 & $4.864[-1]$ & $2.700[+8]$ & 1.11 \\
\hline $5 s 5 g{ }^{3} G_{3}$ & $4 f 5 s{ }^{1} F_{3}^{\circ}$ & 94503 & $8.702[-2]$ & $7.406[+7]$ & 1.32 \\
\hline $5 s 5 g^{3} G_{4}$ & $4 f 5 s{ }^{1} F_{3}^{\circ}$ & 95131 & $5.855[-1]$ & $3.927[+8]$ & 0.89 \\
\hline $5 s 5 g{ }^{1} G_{4}$ & $4 f 5 s{ }^{1} F_{3}^{\circ}$ & 96417 & $2.195[+0]$ & $1.513[+9]$ & 0.94 \\
\hline $5 s 5 g^{3} G_{3}$ & $5 p 5 d^{3} F_{3}^{\circ}$ & 51025 & $4.749[-2]$ & $1.178[+7]$ & 0.90 \\
\hline $5 s 5 g{ }^{3} G_{4}$ & $5 p 5 d^{3} F_{3}^{\circ}$ & 51653 & $6.797[-1]$ & $1.344[+8]$ & 0.70 \\
\hline $5 s 5 g{ }^{1} G_{4}$ & $5 p 5 d^{3} F_{3}^{\circ}$ & 52939 & $8.157[-2]$ & $1.694[+7]$ & 0.67 \\
\hline $5 s 6 d^{3} D_{3}$ & $4 f 5 s{ }^{3} F_{4}^{\circ}$ & 66476 & $4.647[-1]$ & $1.957[+8]$ & 1.12 \\
\hline $5 s 5 g{ }^{1} G_{4}$ & $4 f 5 s{ }^{3} F_{4}^{\circ}$ & 99690 & $5.445[-2]$ & $4.011[+7]$ & 0.93 \\
\hline $5 s 5 g^{3} G_{5}$ & $4 f 5 s{ }^{3} F_{4}^{\circ}$ & 99632 & $3.011[+0]$ & $1.812[+9]$ & 0.92 \\
\hline $5 s 5 g{ }^{3} G_{4}$ & $5 p 5 d^{3} F_{4}^{\circ}$ & 44954 & $9.296[-3]$ & $1.392[+6]$ & 1.22 \\
\hline $5 s 5 g{ }^{3} G_{5}$ & $5 p 5 d d^{3} F_{4}^{\circ}$ & 46181 & $3.671[-1]$ & $4.748[+7]$ & 0.64 \\
\hline
\end{tabular}

